\title{
ERK5 is activated by oncogenic BRAF and promotes melanoma growth
}

\author{
Ignazia Tusa ${ }^{1}$ - Sinforosa Gagliardi ${ }^{2}$ - Alessandro Tubita ${ }^{1}$ Silvia Pandolfi, ${ }^{2,3}$. Carmelo Urso ${ }^{4} \cdot$ Lorenzo Borgognoni $^{5}$. \\ Jinhua Wang ${ }^{6,7} \cdot$ Xianming Deng ${ }^{6,7,8} \cdot$ Nathanael S Gray $\mathbb{1}^{6,7} \cdot$ Barbara Stecca $\mathbb{D}^{2} \cdot$ Elisabetta Rovida $\mathbb{1}^{1}$
}

Received: 27 September 2017 / Revised: 21 December 2017 / Accepted: 17 January 2018 / Published online: 27 February 2018

(c) The Author(s) 2018. This article is published with open access

\begin{abstract}
Malignant melanoma is among the most aggressive cancers and its incidence is increasing worldwide. Targeted therapies and immunotherapy have improved the survival of patients with metastatic melanoma in the last few years; however, available treatments are still unsatisfactory. While the role of the BRAF-MEK1/2-ERK1/2 pathway in melanoma is well established, the involvement of mitogen-activated protein kinases MEK5-ERK5 remains poorly explored. Here we investigated the function of ERK5 signaling in melanoma. We show that ERK5 is consistently expressed in human melanoma tissues and is active in melanoma cells. Genetic silencing and pharmacological inhibition of ERK5 pathway drastically reduce the growth of melanoma cells and xenografts harboring wild-type (wt) or mutated BRAF (V600E). We also found that oncogenic BRAF positively regulates expression, phosphorylation, and nuclear localization of ERK5. Importantly, ERK5 kinase and transcriptional transactivator activities are enhanced by BRAF. Nevertheless, combined pharmacological inhibition of BRAFV600E and MEK5 is required to decrease nuclear ERK5, that is critical for the regulation of cell proliferation. Accordingly, combination of MEK5 or ERK5 inhibitors with BRAFV600E inhibitor vemurafenib is more effective than single treatments in reducing colony formation and growth of BRAFV600E melanoma cells and xenografts. Overall, these data support a key role of the ERK5 pathway for melanoma growth in vitro and in vivo and suggest that targeting ERK5, alone or in combination with BRAF-MEK1/2 inhibitors, might represent a novel approach for melanoma treatment.
\end{abstract}

These authors contributed equally: Ignazia Tusa, Sinforosa Gagliardi and Alessandro Tubita.

Co-last authors: Barbara Stecca and Elisabetta Rovida.

Electronic supplementary material The online version of this article (https://doi.org/10.1038/s41388-018-0164-9) contains supplementary material, which is available to authorized users.

Barbara Stecca

barbara.stecca@ittumori.it

$\triangle$ Elisabetta Rovida

erovida@unifi.it

1 Department of Clinical and Experimental Biomedical Sciences, University of Florence, Florence, Italy

2 Core Research Laboratory - Istituto Toscano Tumori, Florence, Italy

3 Present address: VIB-KU Leuven Center for Cancer Biology, Leuven, Belgium

\section{Introduction}

Malignant melanoma is one of the most aggressive types of cancer. While early-stage melanoma can be cured in the majority of cases by surgical excision, late-stage melanoma is a highly lethal disease $[1,2]$. Common genetic alterations associated with melanoma include mutually exclusive mutations in BRAF (50-60\%), NRAS (20-25\%), and NF1

4 Anatomic Pathology Unit, Dermatopathology Section, S.M. Annunziata Hospital, Florence, Italy

5 Plastic Surgery Unit, Regional Melanoma Referral Center, S.M. Annunziata Hospital, Florence, Italy

6 Department of Cancer Biology, Dana-Farber Cancer Institute, Boston, MA, USA

7 Department of Biological Chemistry and Molecular Pharmacology, Harvard Medical School, Boston, MA, USA

8 Present address: State Key Laboratory of Cellular Stress Biology, Innovation Center for Cell Signaling Network, School of Life Sciences, Xiamen University, Xiamen, Fujian 361102, China 


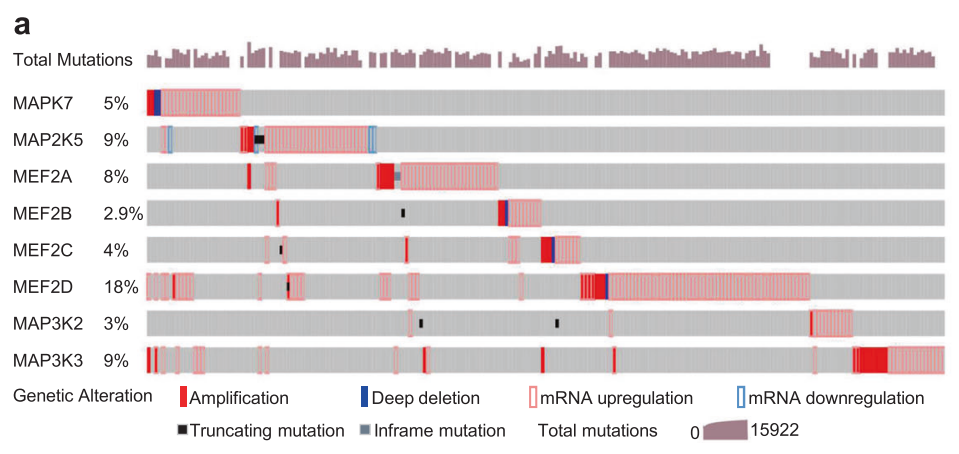

c

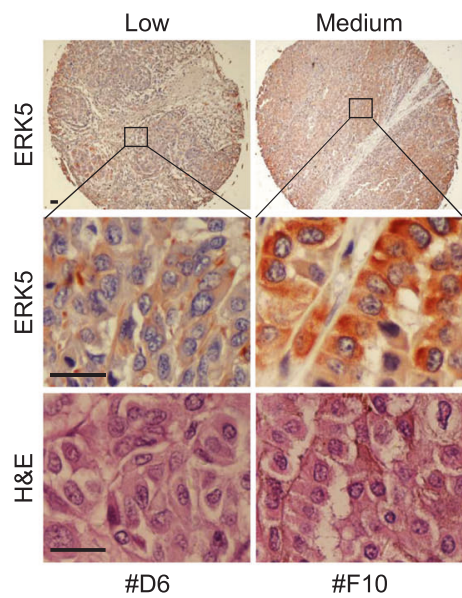

e

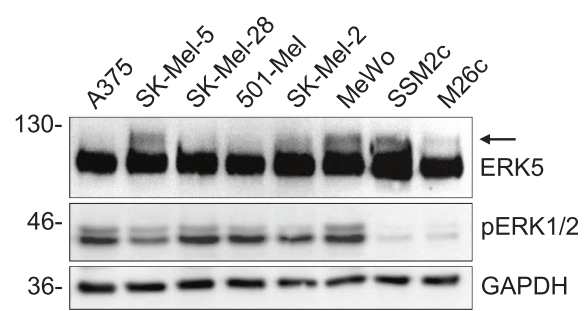

f

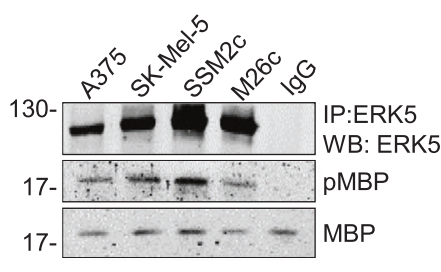

b

Cases w/o ERK5 alterations

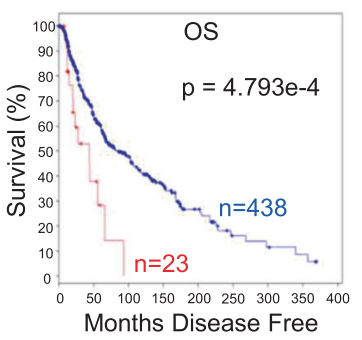

$\square$ Cases w/ ERK5 alterations

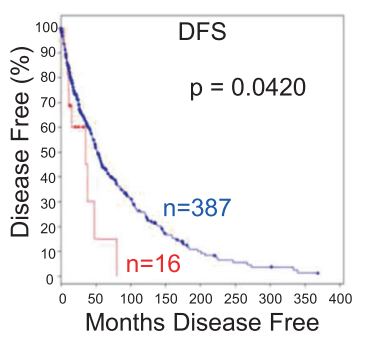

d

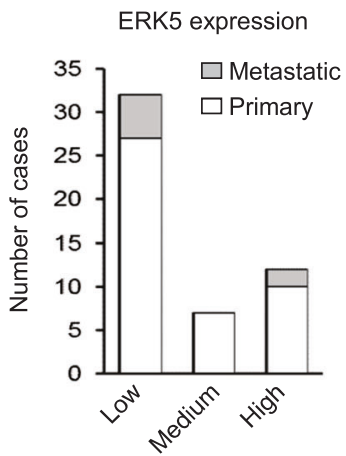

Fig. 1 Expression of ERK5 in melanoma. a Genomic profiles of components of the ERK5 pathway in melanoma patients obtained from Skin Cutaneous Melanoma data set (TCGA, Provisional) using cBioportal database (www.cbioportal.org) [28, 29]. Shown are 223 out of $479(47 \%)$ primary and metastatic melanomas with gene copy number and mRNA alterations. Putative passenger mutations are not included. MAPK7 and MAP2K5 are the genes encoding for ERK5/ BMK1 or MEK5, respectively. b Kaplan-Meier overall survival (OS) and disease-free survival (DFS) in melanoma patients with (red) or without (blue) ERK5 genetic alterations (data set from cBioportal restricted to "AMP EXP > =2" to exclude patients harboring MAPK7 deletion). Median months survival: 43.8 vs $85(p=4.79 \mathrm{e}-4$, log-rank test); median months disease free: 35 vs $51.5(p=0.042$, log-rank test). c Immunohistochemical analysis of ERK5 (top and middle panels) and H\&E (bottom panels) in human melanoma tissue array cores. ERK5 was counterstained with hematoxylin. Samples were

$(14 \%)[3,4]$. These mutations drive the hyperactivation of the mitogen-activated protein kinases (MAPK) extracellular signal-regulated kinase 1 and 2 (ERK1/2) [5], which, in turn, promotes tumor cell growth. scored as low, medium, or high ERK5 expression (see Supplementary Table 1 for details and clinico-pathological characteristics of samples). Top panels show representative cores at low magnification. Middle and bottom panels are high magnification of top images. Bar $=40 \mu \mathrm{m}$. d Quantification of ERK5 expression in primary and metastatic melanomas from c. e Expression of ERK5 in melanoma cell lines. Cells were serum starved for $24 \mathrm{~h}$ and total cell lysates were obtained. GAPDH was used as a loading control. Arrow indicates ERK5 slow migrating band. f In vitro kinase assay from immunoprecipitated ERK5 in melanoma cell lines. IgG is a control sample without antiERK5 antibody. MBP was used as a loading control. g Expression of ERK5 in nuclear and cytoplasmic extracts in four melanoma cell lines. Black lines indicate that samples were run on the same gel but were noncontiguous. GAPDH and Fibrillarin were used as cytoplasmic or nuclear markers, respectively. e-g Representative blots from three independent experiments

Over the last few years, new BRAF-targeting and MEK1/2-targeting drugs as well as immunotherapy have improved progression-free and overall survival of melanoma patients [6-8]. However, development of resistance in 
patients with oncogenic mutations remains a major obstacle to the long-term clinical benefit of targeted therapies [9]. In contrast, immunotherapy is the only effective treatment option for patients who are wild type (wt) for BRAF, NRAS, or NF1, albeit some of these patients fail to respond to immunotherapy [10]. Thus, there is an urgent need to identify druggable signaling pathways critical for melanoma cell growth.

A further member of the MAPK family, ERK5 (also referred to as big mitogen-activated Kinase 1, BMK1), is involved in cell survival, anti-apoptotic signaling, angiogenesis, differentiation and proliferation of several cell types [11]. ERK5 possesses an N-terminal kinase domain highly homologous to that of ERK1/2 and a unique long Cterminal domain. Mitogens that activate receptor tyrosine kinases [12], as well as cytokines and stress factors may lead to the activation of MAP3K2 and MAP3K3, upstream activators of MEK5, which in turn activates ERK5 through phosphorylation on Thr218/Tyr220 in the catalytic domain $[11,13]$. Moreover, extensive phosphorylation of ERK5 at the $\mathrm{C}$ terminus may occur during cell cycle progression in a MEK5-independent manner [14, 15]. Phosphorylation at MEK5 consensus site and/or at $\mathrm{C}$ terminus as well as other mechanisms are involved in ERK5 nuclear translocation, which is a key event for the regulation of cell proliferation [16-19]. The MEK5-ERK5 pathway is involved in the pathogenesis of different types of cancer, including highly aggressive forms of breast [20,21] and prostate cancer [22], hepatocellular carcinoma [23] and multiple myeloma [24]. Thus, the MEK5-ERK5 pathway is becoming a promising target for cancer treatment $[25,26]$. Here we explored the role of ERK5 pathway in melanoma and its regulation by oncogenic BRAF.

\section{Results}

\section{ERK5 is consistently expressed and active in human melanoma}

In silico data analysis of components of ERK5 signaling indicated that the activators MAP3K2, MAP3K3 and MAP2K5 (alternative name for MEK5), MAPK7 itself (the gene encoding for ERK5), and the downstream targets MEF2 transcription factors [27] (i.e., MEF2A, MEF2B, $\mathrm{MEF} 2 \mathrm{C}$, and MEF2D) are altered (mutations, gene copy number, or mRNA alterations) in $47 \%$ of human melanomas (Fig. 1a) [28, 29]. Interestingly, melanoma patients with MAPK7 alterations (mRNA upregulation and MAPK7 amplifications but not deletions) showed reduced diseasefree survival ( $p=0.042)$ and a trend toward shorter overall survival ( $p=4.793 \mathrm{e}-4)$ compared to patients without such alterations (Fig. 1b). Immunohistochemical analysis of
ERK5 in tissue microarrays showed that ERK5 was expressed in benign and malignant melanocytes, but not in keratinocytes. ERK5 staining was observed in both cytoplasm and nucleus (Fig. 1c). As expected, endothelial cells were positive for ERK5 [11] (Fig. 1c). ERK5 was expressed at low, medium, or high levels in the majority of nevi (9 out of $11 ; 82 \%)$ and of primary and metastatic melanomas (51 out of 66; 77\%; Fig. 1d and Supplementary Table 1). Accordingly, ERK5 was expressed in a number of commercial and patient-derived melanoma cell lines and normal human epidermal melanocytes (NHEM; Supplementary Table 2; Fig. 1e and Supplementary Figure 1a) [30]. Moreover, in most of the cell lines analyzed (SK-Mel-5, SK-Mel-2, MeWo, SSM2c, and M26c) ERK5 presented a slower migrating band, consistent with the phosphorylation (Fig. 1e). As expected, marked ERK1/2 phosphorylation was found in melanoma cells with mutations in BRAF, NRAS, or NF1 (Fig. 1e and Supplementary Table 2). For subsequent experiments, we used two melanoma cell lines expressing wt (SSM2c and M26c) and two harboring mutated (V600E) BRAF (A375 and SK-Mel-5). Kinase assay from immunoprecipitated ERK5 showed that ERK5 is constitutively active in all four cell lines, as shown by phosphorylation of the myelin basic protein (MBP) with respect to the IgG control (Fig. 1f).

ERK5 nuclear translocation is a key event for ERK5 activity [19]. Therefore, we evaluated ERK5 intracellular localization and found that ERK5 was located in both the cytoplasm and the nucleus in all four melanoma cell lines (Fig. 1g). Altogether, these data indicate that the ERK5 pathway is consistently expressed and is active in human melanoma.

\section{ERK5 is required for melanoma cell proliferation in vitro and xenograft growth}

ERK5 plays a relevant role in the growth of several types of cancer [20-26]. Thus, we investigated the effects of the inhibition of ERK5 signaling in melanoma cell proliferation. Genetic inhibition of ERK5 [23] using two specific shRNAs (LV-shERK5-1 and LV-shERK5-2) markedly reduced the growth of melanoma cells harboring wt or mutated (V600E) BRAF (Fig. 2a-c). To investigate whether ERK5 regulates melanoma growth in vivo, A375 and SSM2c melanoma cells stably transduced with LV-c or LVshERK5-1 were subcutaneously injected into athymic nude mice and tumor growth was monitored. ERK5 silencing drastically reduced A375 xenograft growth and diminished by $70 \%$ that of SSM2c xenografts compared to LV-c (Fig. 2d-g). Silencing of ERK5 reduced, but did not abolish, tumor take and delayed tumor appearance in both cell types (Fig. 2h). Western blot analysis in tumors dissected at the end of experiments confirmed the drastic reduction of 
Fig. 2 Silencing of ERK5 inhibits melanoma cell proliferation in vitro and in human melanoma xenografts. a, b Growth curves of A375 (a) and SSM2c (b) cells transduced with LV-shERK5 or LV-c lentiviruses. Data shown are mean \pm SD of one representative experiment out of three performed in triplicates. ${ }^{* *} p<$ 0.01 as determined by Student's $t$-test. c Western blot shows ERK5 silencing efficiency (upper panel). Tubulin was used as loading control. Images of one representative experiment out of three performed with A375 cells transduced with the indicated lentiviruses stained with crystal violet after 10 days (lower panel). d, e In vivo tumor growth after subcutaneous injection of $1 \times 10^{4} \mathrm{~A} 375$ (d) or SSM2c (e) melanoma cells transduced with LV-c or LVshERK5-1 lentiviruses. Data shown are mean \pm SEM. $* p<$ $0.05, * * p<0.01$ and $* * * p<$ 0.001 as determined by Student's $t$-test. f, g Representative images of A375 and SSM2c xenografts in athymic nude mice. Bar = $10 \mathrm{~mm}$. $\mathbf{h}$ Table shows tumor take (number of tumors formed per number of injections) and latency (time from injection to tumor measurability) for each group. Data shown are mean \pm SEM. i Western blot analysis of representative tumors derived from A375 and SSM2c xenografts. Actin was used as loading control a

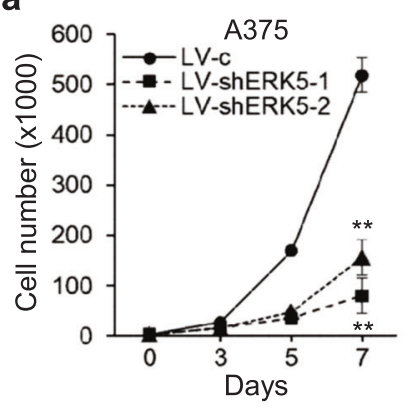

b

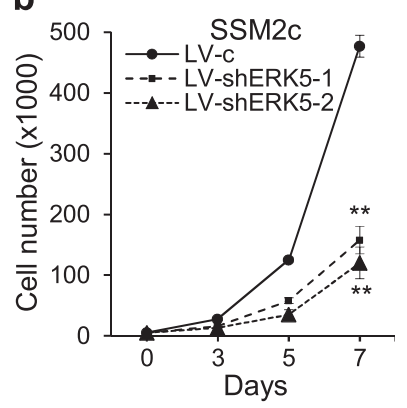

C

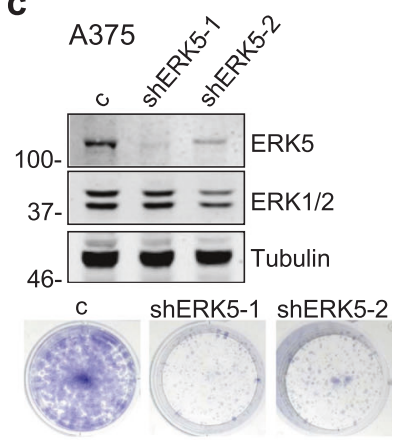

d

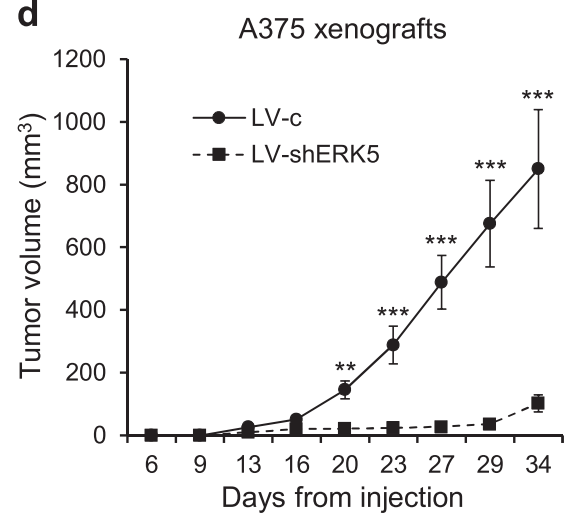

f

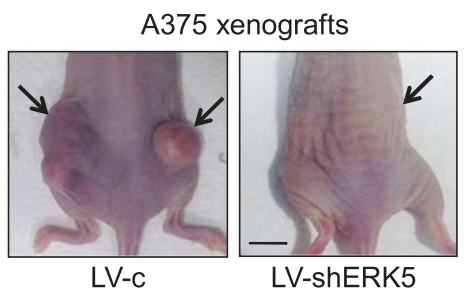

h

\begin{tabular}{|lcc|}
\hline & Tumor take & Latency (days) \\
\hline A375 LV-c & $11 / 12$ & $13.8 \pm 0.4$ \\
A375 LV-shERK5 & $5 / 12$ & $20 \pm 3.1$ \\
SSM2c LV-c & $10 / 13$ & $25.9 \pm 2.3$ \\
SSM2c LV-shERK5 & $7 / 12$ & $31 \pm 3.7$ \\
\hline
\end{tabular}

e

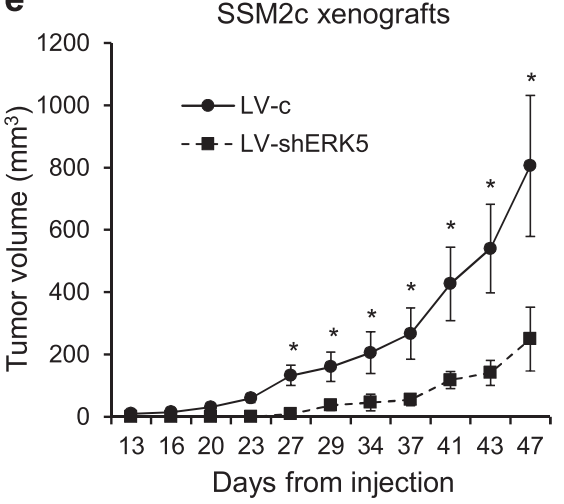

g

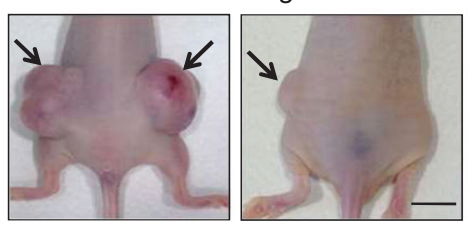

LV-shERK5

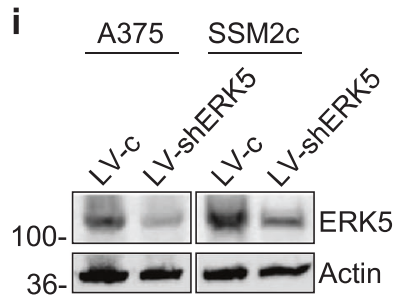

ERK5 in LV-shERK5-transduced cells (Fig. 2i). Altogether, these results indicate that ERK5 plays a critical role in melanoma cell growth in vitro and in vivo.

\section{Pharmacological inhibition of the ERK5 pathway decreases melanoma cell growth}

In view of a possible translation to the clinics, we determined the effects of pharmacological inhibition of the ERK5 pathway using the ERK5 inhibitor XMD8-92 [31] or the MEK5 inhibitor BIX02189 [32]. Either drug abolished the slower migrating band of ERK5, proving reduced ERK5 phosphorylation likely as a consequence of reduced autophosphorylation (XMD8-92) or decreased interaction with and phosphorylation by MEK5 (BIX02189) (Supplementary Figure 1b). XMD8-92 or BIX02189 treatment decreased the number of viable cells in a dose-dependent manner in several melanoma cell lines expressing either wt or BRAFV600E (Fig. 3a-c), with IC50 values ranging from 2.3 to $3.7 \mu \mathrm{M}$ for XMD8-92, and from 5.4 to $7.1 \mu \mathrm{M}$ for BIX02189. Moreover, because XMD8-92 has been reported to be a dual ERK5/BRD4 inhibitor [33], we confirmed the effects on cell proliferation with JWG0-45 (Supplementary Figure 2a), a novel ERK5 inhibitor with much lower affinity 
a

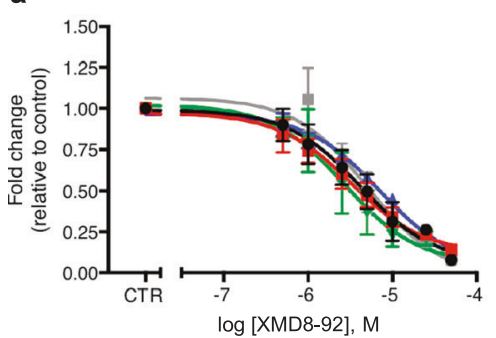

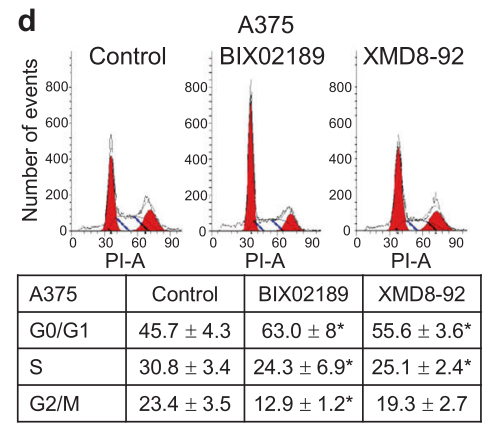

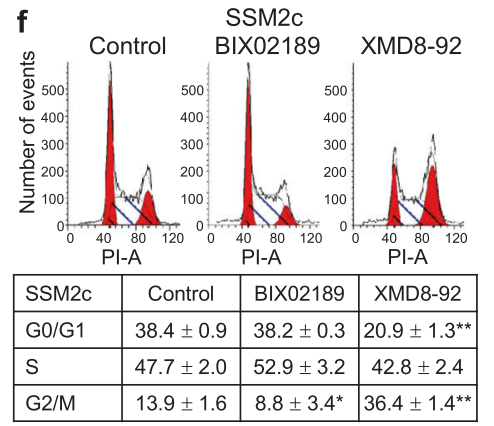

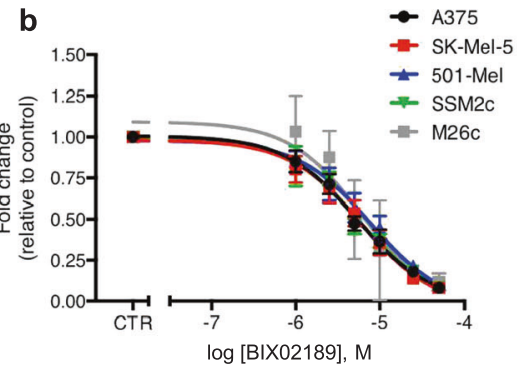

e
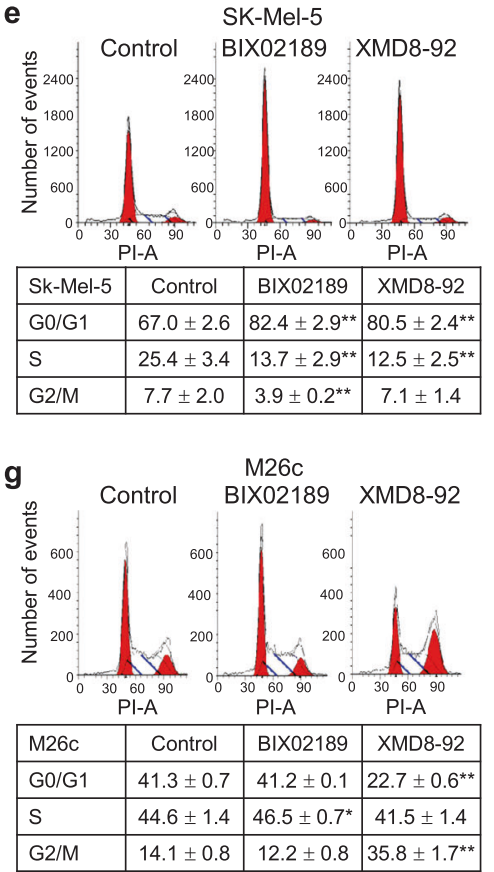

C

\begin{tabular}{|l|c|c|c|}
\hline & $\begin{array}{c}\text { XMD8-92 } \\
\text { IC50 }(\mu \mathrm{M})\end{array}$ & $\begin{array}{c}\text { BIX02189 } \\
\text { IC50 }(\mu \mathrm{M})\end{array}$ & $\begin{array}{c}\text { Vem } \\
\text { IC50 }(\mathrm{nM})\end{array}$ \\
\hline SSM2C & $2.3 \pm 0.5$ & $5.5 \pm 0.7$ & n.d. \\
\hline M26c & $2.9 \pm 0.4$ & $6.3 \pm 0.8$ & n.d. \\
\hline A375 & $2.6 \pm 0.7$ & $5.4 \pm 0.4$ & $58.9 \pm 0.7$ \\
\hline SK-Mel-5 & $2.3 \pm 0.5$ & $6.1 \pm 0.6$ & $65.2 \pm 0.5$ \\
\hline 501-Mel & $3.7 \pm 0.5$ & $7.1 \pm 0.8$ & $57 \pm 0.5$ \\
\hline
\end{tabular}

h A375 SK-Mel-5 SSM2c M26c

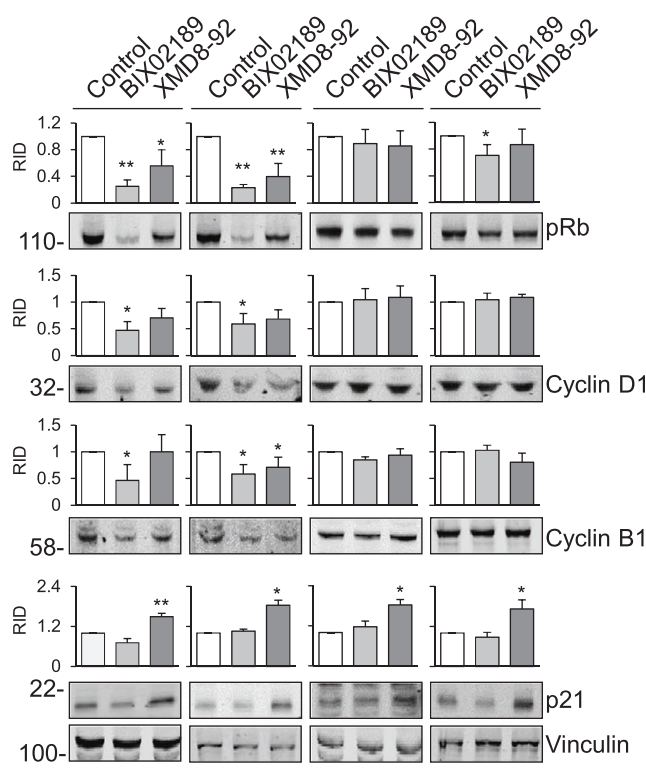

Fig. 3 Pharmacological inhibition of the ERK5 pathway reduces melanoma cell proliferation in vitro. a, b Dose-response curves of XMD8-92 (a) and BIX02189 (b) in a number of melanoma cell lines after $72 \mathrm{~h}$ of treatment or DMSO (CTR). Data shown are mean \pm SD from four independent experiments performed in triplicates. c IC50 values for XMD8-92, BIX02189 and vemurafenib in melanoma cells treated for $72 \mathrm{~h}$. d-g Cell cycle phase distribution plots and values (tables) of BRAFV600E-expressing cells (A375 and SK-Mel-5) (d, e) and of wt BRAF cells (SSM2c and M26c) (f, g). Cells were treated for

toward BRD proteins [34]. Furthermore, no reduction in cell proliferation was observed when XMD8-92 (up to 5 $\mu \mathrm{M}$, which is the concentration used throughout the manuscript) was applied to ERK5-silenced melanoma cells (Supplementary Figure $2 \mathrm{~b}$ and $\mathrm{c}$ ). These results seem to exclude that the effect of XMD8-92 on cell proliferation, at least at the concentration used in these cells, are due to offtarget effects.

In order to analyze how inhibition of the ERK5 pathway affects cell growth, we performed cell cycle analysis. In BRAFV600E-expressing cells (A375 and SK-Mel-5), treatment with either XMD8-92 or BIX02189 markedly increased the fraction of cells in G0/G1 phase, mainly at the expenses of those in $S$ phase. In addition, treatment with
$48 \mathrm{~h}$ with XMD8-92 $(5 \mu \mathrm{M})$ or BIX02189 $(10 \mu \mathrm{M})$. Data shown are mean \pm SD from four independent experiments. $* p<0.05, * * p<0.01$ as determined by Student's $t$-test. h Expression or phosphorylation status of cell cycle regulators in cells treated for $24 \mathrm{~h}$ with XMD8-92 $(5 \mu \mathrm{M})$ or BIX02189 $(10 \mu \mathrm{M}) . * p<0.05, * * p<0.01$ as determined by Student's $t$-test. Total cell lysates were obtained. Vinculin was used as loading control. Densitometric analysis was performed on three independent experiments. Controls were treated with DMSO

BIX02189 significantly reduced the number of cells in G2/ M phase (Fig. 3d, e). Consistently, BIX02189 and, to a lesser extent, XMD8-92 reduced the level of pRb (Ser807) in BRAFV600E-expressing cells (Fig. 3h). Treatment with XMD8-92 increased the expression of the cyclin-dependent kinase inhibitor (CDKi) p21. Finally, treatment with BIX02189 and, to a lesser extent, XMD8-92 decreased the expression of cyclin D1 and B1 (Fig. 3h).

In wt BRAF melanoma cells (SSM2c and M26c), XMD8-92 treatment resulted in a marked accumulation in G2/M phase at the expense of those in G0/G1 and $S$ phases (Fig. 3f, g) and increased p21 expression. JWG-045 produced similar effect on cell cycle phase distribution (Supplementary Figure 2d). On the other hand, 

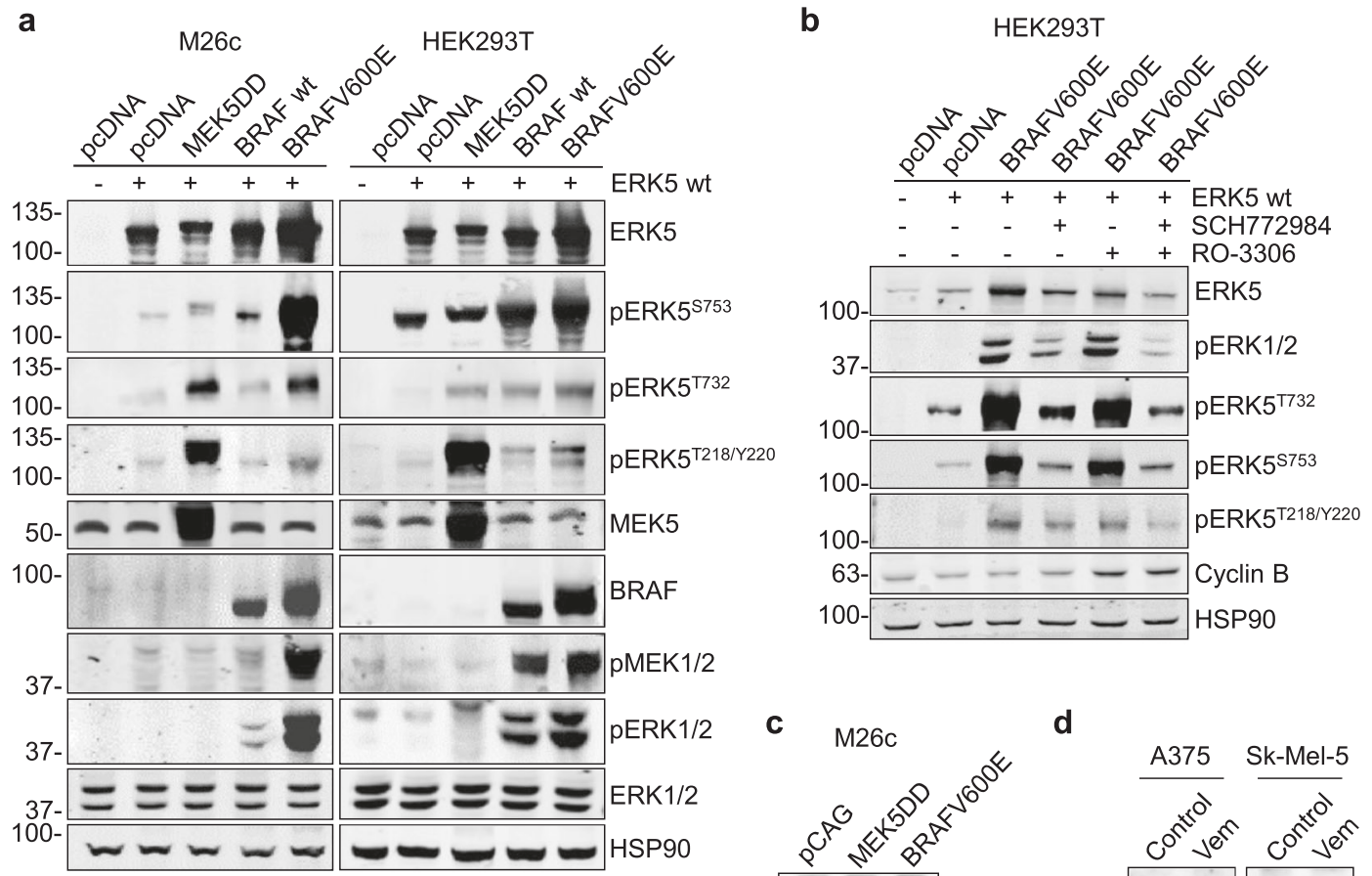

C

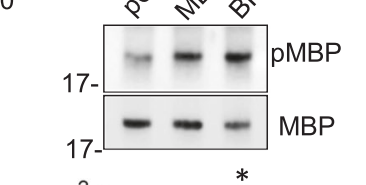

d

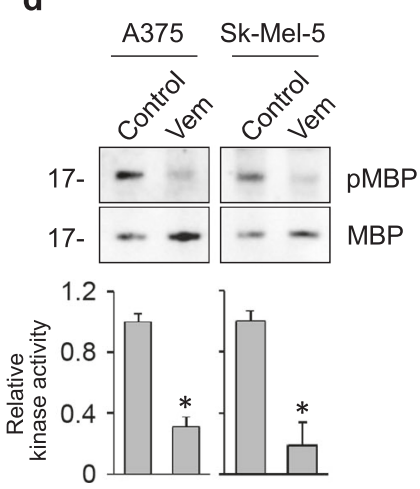

Fig. 4 Oncogenic BRAF enhances ERK5 expression and activation. a M26c melanoma cells and HEK-293T cells were transfected with equimolar amounts of pcDNA (control, -) or wt ERK5 in combination with pcDNA, constitutively active MEK5 (MEK5DD), wt BRAF, or BRAFV600E plasmids. Cells were lysed after $48 \mathrm{~h}$ and western blot was performed with the indicated antibodies. HSP90 was used as a loading control. b HEK-293T cells were transfected with equimolar amounts of pcDNA (control) or wt ERK5 in combination with pcDNA or BRAFV600E plasmids. Cells were treated with the ERK1/2 inhibitor SCH772984 $(0.5 \mu \mathrm{M})$ and/or the CDK1 inhibitor RO-3306 (9 $\mu \mathrm{M})$ during the last $18 \mathrm{~h}$ of transfection. Cells were lysed after $24 \mathrm{~h}$ and western blot was performed with the indicated antibodies. HSP90 was

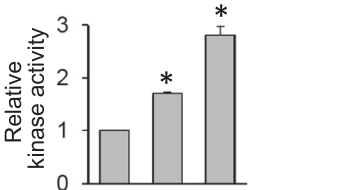

used as a loading control. c In vitro kinase assay for ERK5 immunoprecipitated from M26c cells transfected with equimolar amounts of pCAG, constitutively active MEK5 (MEK5DD) or BRAFV600E plasmids. MBP was used as a loading control. d In vitro kinase assay for ERK5 immunoprecipitated from A375 or SK-Mel-5 cells treated with $1 \mu \mathrm{M}$ vemurafenib (Vem) or DMSO (Control) for $24 \mathrm{~h}$. MBP was used as a loading control. Blots are representative images from five (a) or three (b) independent experiments. c-d Relative ERK5 kinase activity determined by densitometric quantification of pMBP normalized for MBP from three independent experiments (mean \pm SD). pCAG and DMSO controls were set to $1 .{ }^{*} p<0.05$ as determined by Student's t-test
BIX02189 slightly affected cell cycle distribution, with a trend toward an increase in the number of cells in $S$ phase and a reduction of those in $\mathrm{G} 2 / \mathrm{M}$ phase (Fig. 3f, g). Accordingly, no major differences were found in the phosphorylation of $\mathrm{Rb}$ nor in the expression of Cyclin $\mathrm{B} 1$ and D1 following XMD8-92 or BIX02189 treatment. The reduction of cell number observed in melanoma cells treated with BIX02189 may be due to an increase of apoptosis (Supplementary Figure 3). These results demonstrated that pharmacological inhibition of the MEK5-ERK5 signaling in vitro mimics the effects of ERK5 genetic inhibition in reducing melanoma cell proliferation.

\section{Oncogenic BRAF increases ERK5 activity}

ERK5 is expressed and active in melanoma cells (Fig. 1). Because BRAF is mutated in $\sim 50 \%$ of melanomas, we investigated whether BRAF modulates ERK5 activation. To address this point, we overexpressed oncogenic BRAF in HEK-293T and melanoma cells expressing wt BRAF. In both cell types, BRAFV600E increased endogenous and exogenous ERK5 protein levels (Supplementary Figure 4 and Fig. 4a). Oncogenic BRAF induced a robust phosphorylation of ERK5 at Ser753 and at Thr732 (Fig. 4a), two residues in the ERK5 C-terminal region that are putative 
a

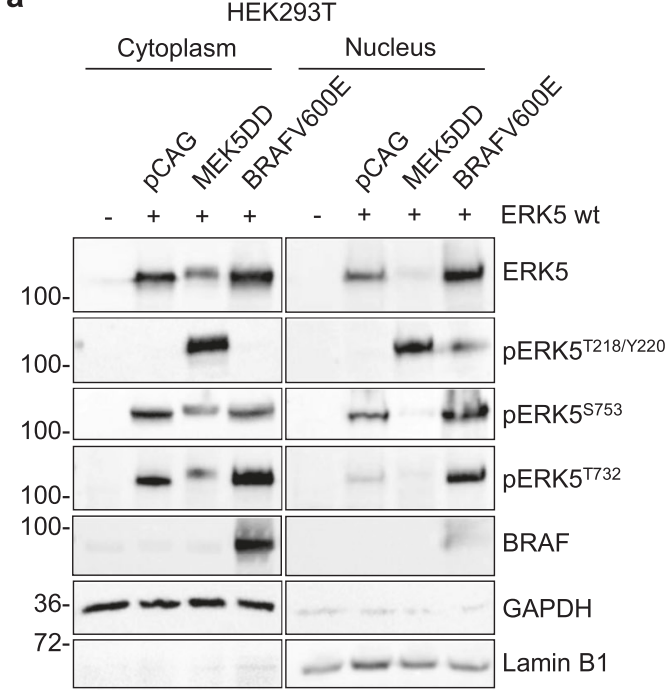

b

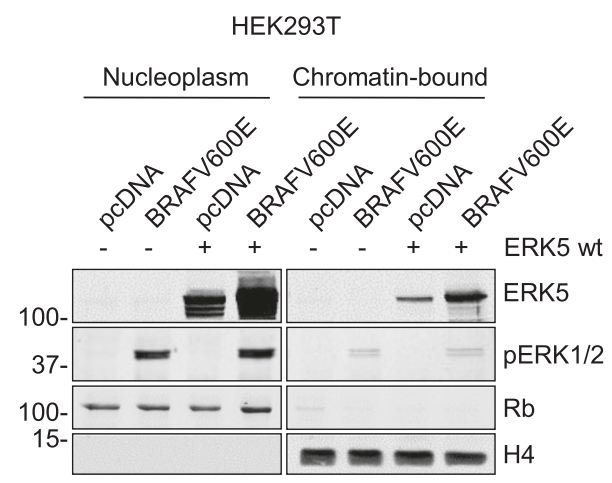

c

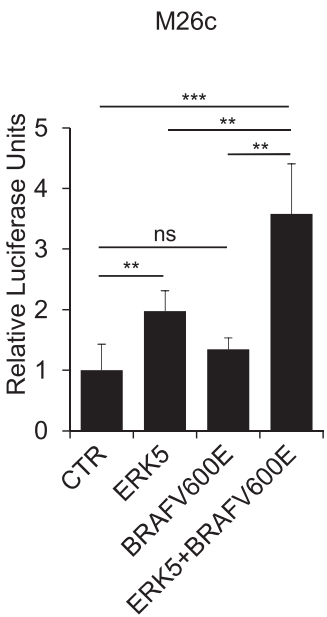

Fig. 5 Oncogenic BRAF increases ERK5 nuclear localization and transcriptional transactivator activity. a Nucleo-cytoplasmic fractionation in HEK-293T cells transfected with equimolar amounts of wt ERK5 in combination with the empty vector pCAG, constitutively active MEK5 (MEK5DD) or BRAFV600E plasmids. BRAFV600E increases the level and the phosphorylation of nuclear ERK5. GAPDH and Lamin B1 were used as cytoplasmic or nuclear markers, respectively. b Nucleoplasm and chromatin-bound fraction from HEK-293T cells transfected with equimolar amounts of empty or BRAFV600E
pcDNA plasmids in presence or not of wt ERK5. Rb and Histone $\mathrm{H} 4$ were used as nucleoplasm or chromatin-bound markers, respectively. (a, b) Representative blots from three independent experiments. c Quantification of dual reporter luciferase assay in M26c melanoma cells showing that BRAFV600E enhances the transcriptional transactivator activity of wt ERK5. Relative luciferase activity was firefly/ Renilla ratios, with the level induced by control equated to 1 . Data represent mean $\pm \mathrm{SD}$ of three independent experiments. ${ }^{* *} p<0.01$, $* * * p<0.001$ as determined using one-way ANOVA autophosphorylation sites as well as targets of CDK1 and/or ERK1/2 (see below) [15, 35]. Ectopic expression of constitutively active MEK5 (MEK5DD) induced robust ERK5 phosphorylation at Thr218/Tyr220, as expected, and increased ERK5 phosphorylation at Thr732 (Fig. 4a). Overexpression of oncogenic BRAF induced ERK5 phosphorylation at Thr218/Tyr220, although to a lower level than that induced by MEK5DD (Fig. 4a). Ectopic expression of wt BRAF elicited similar effects on the expression and phosphorylation of ERK5, although to a lesser extent. These results indicate that overexpression of BRAF, either wt or mutated (V600E), determines an increase of ERK5 protein level and phosphorylation at different residues.

To assess whether phosphorylation of ERK5 at the C terminus is critical for the regulation of ERK5 expression levels by BRAF, we used a mutated form of ERK5 (ERK5 $\Delta 713$ ) lacking the $\mathrm{C}$ terminus. BRAFV600E was able to enhance the expression level of ERK5 $\Delta 713$ at the same level of full-length ERK5, possibly due to higher phosphorylation at Thr218/Tyr220 with respect to wt ERK5 (Supplementary Figure 5). Nevertheless, BRAFV600E increased the level of expression of ERK5-AEF (a mutated form of ERK5 that cannot be phosphorylated at Thr218/ Tyr220), indicating that the regulation of ERK5 expression level by BRAFV600E likely depends on phosphorylation at any of the aforementioned residues. This is well in keeping with the fact that phosphorylation at different residues has been reported to sustain ERK5 protein levels [18].

Because Ser753 can be also phosphorylated by CDK1 (ref. 15), whereas Thr732 is phosphorylated by both CDK1 and ERK1/2 (ref. 35), we investigated the involvement of these two kinases in BRAFV600E-dependent regulation of ERK5. Treatment with the ERK1/2 inhibitor SCH772984 or the CDK1 inhibitor RO-3306 reduced ERK5 protein level and phosphorylation at Ser753 and Thr732, and their combination completely abolished BRAFV600E-induced effects (Fig. 4b). The efficacy of SCH772984 on ERK1/2 and of RO-3306 on CDK1 was demonstrated, respectively, by decreased phosphorylation of ERK1/2 and increase of Cyclin B, as previously reported [15]. These data indicate that CDK1 and ERK1/2 contribute to BRAF-dependent regulation of ERK5 phosphorylation and protein amount.

We next investigated whether oncogenic BRAF affects ERK5 activity. Overexpression of BRAFV600E enhanced ERK5 kinase activity (Fig. 4c). Consistently, pharmacological inhibition of BRAFV600E with vemurafenib markedly reduced basal ERK5 kinase activity in A375 and SKMel-5 cells (Fig. 4d). To exert its proliferative activity, ERK5 translocates into the nucleus [19]. Overexpression of BRAFV600E increased the nuclear amount of total and phosphorylated (at Ser753 and Thr732) ERK5 (Fig. 5a). More importantly, oncogenic BRAF increased the amount 
a

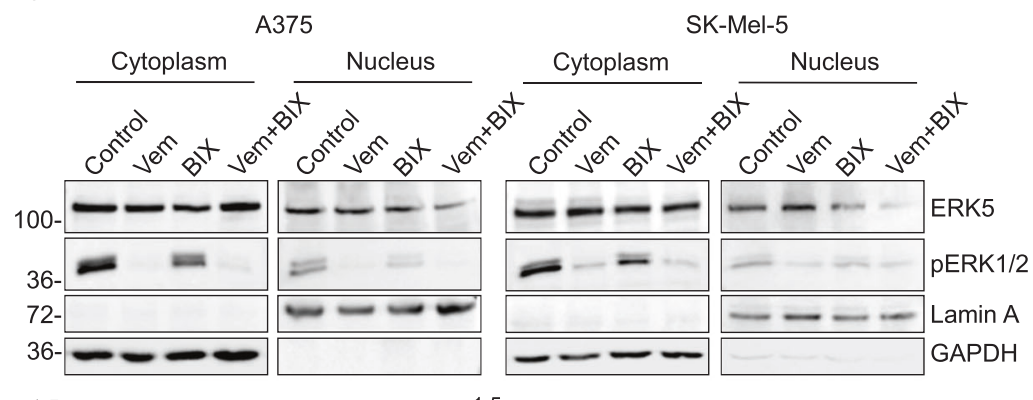

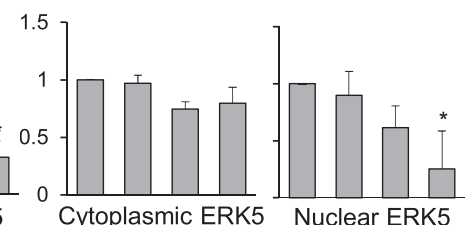

b
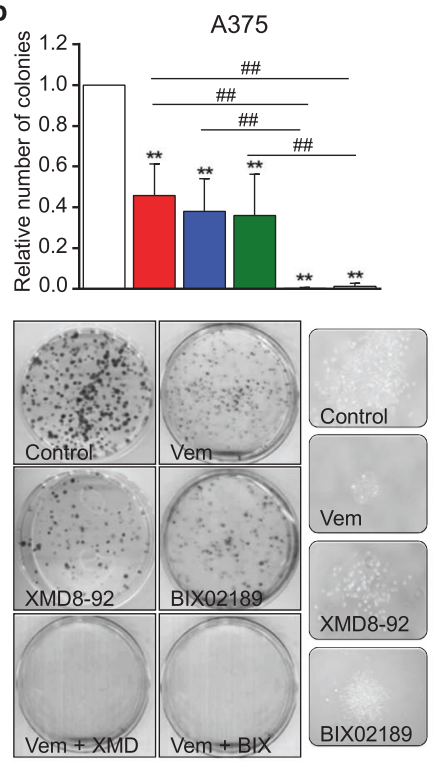

c
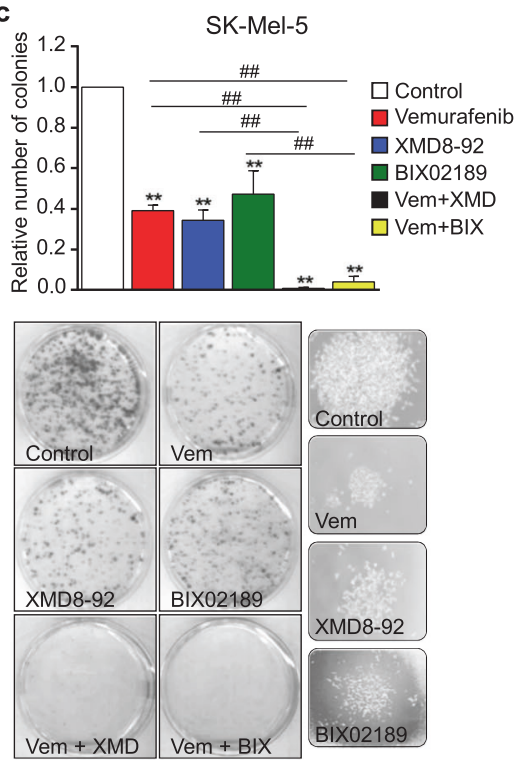

d

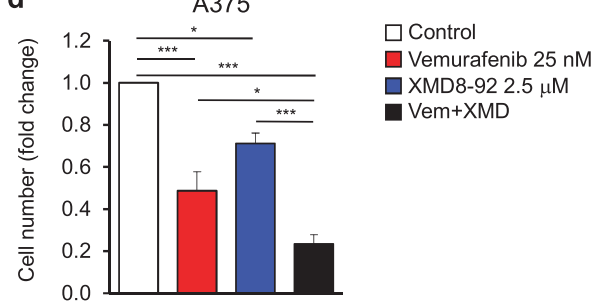

e

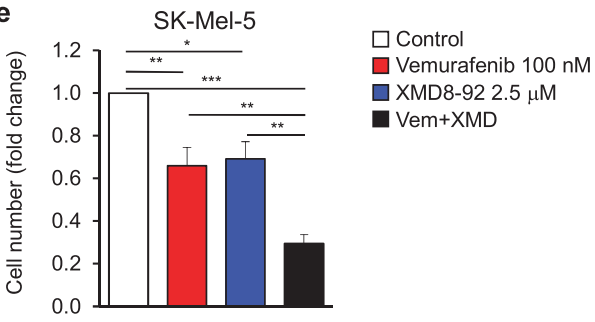

f

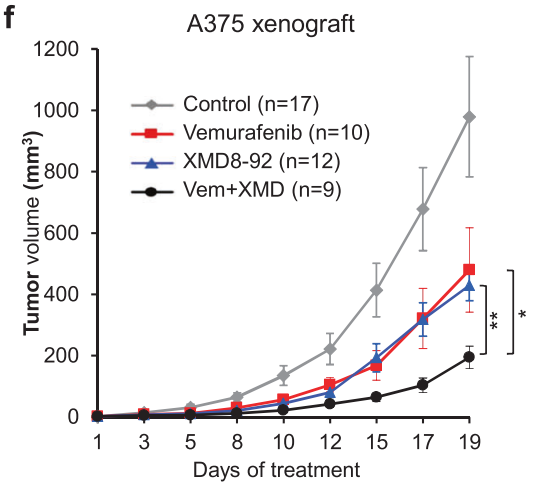

g

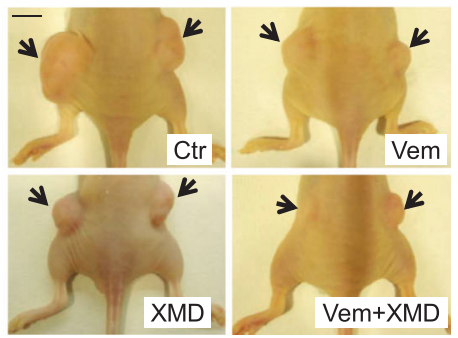

Fig. 6 Combined pharmacological inhibition of the ERK5 pathway and BRAFV600E reduces melanoma cell growth in vitro and in vivo. a Cytoplasmic and nuclear extracts of A375 or SK-Mel-5 melanoma cells showing the effect of vemurafenib $(1 \mu \mathrm{M})$, BIX02189 $(10 \mu \mathrm{M})$ or the combination on the expression of endogenous ERK5. GAPDH and Lamin A were used as cytoplasmic or nuclear markers, respectively. Quantification of cytoplasmic and nuclear endogenous ERK5 normalized for loading control from three independent experiments is shown in histograms (mean $\pm \mathrm{SD}$ ). $* p<0.05$ as determined by Student's $t$-test. b, c 2D colony forming assay in A375 (b) and SK-Mel-5 (c) cells treated with DMSO (Control) or IC50 concentration of XMD8-92 (A375: $2.5 \mu \mathrm{M}$; SK-Mel-5: $2.0 \mu \mathrm{M}$ ), BIX02189 (A375: 2.5 $\mu \mathrm{M}$; SK-Mel-5: $5.5 \mu \mathrm{M}$ ) or vemurafenib (A375: $0.5 \mu \mathrm{M}$; SK-Mel-5: $0.45 \mu \mathrm{M})$ alone or in combination. Histograms represent mean \pm SD from four independent experiments. $* * p<0.01$ treatment vs Control; ${ }^{\# \#} p<0.01$ between indicated samples. $P$ values were determined using one-way ANOVA. Below are representative images of plates (left) or colonies (right). d, e Number of viable A375 (d) and SK-Mel-5 (e) cells treated for $72 \mathrm{~h}$ with DMSO (Control), vemurafenib, XMD8-92, or the combination (Vem + XMD) at the indicated concentrations. Histograms represent mean \pm SD from one representative experiment out of three performed in triplicate. Bliss independence indicates additive effects in Vem + XMD vs Vem or XMD. * $p<0.05$, ** $p<$ $0.01, * * * p<0.001$ as determined by one-way ANOVA. f In vivo tumor growth of A375 melanoma cells subcutaneously injected $(1 \times$ $\left.10^{4}\right)$. Mice were treated at tumor appearance with vemurafenib $(20 \mathrm{mg} /$ $\mathrm{kg})$, XMD8-92 $(25 \mathrm{mg} / \mathrm{kg})$ or the combination. After 19 days of treatment mice were sacrificed. Data shown are mean \pm SEM. Combined treatment increased the efficacy of XMD8-92 or vemurafenib alone. Number of tumors for each group is indicated. $* p \leq 0.05$; ** $p<$ 0.01 as determined by one-way ANOVA. $\mathbf{g}$ Representative images of A375 xenografts in athymic nude mice. $B a r=10 \mathrm{~mm}$ 
of ERK5 in the chromatin-bound fraction (Fig. 5b) and enhanced the ability of ERK5 to induce transcription using a MEF2 luciferase reporter (Fig. 5c). Altogether, these data indicate that oncogenic BRAF positively regulates ERK5 activity.

\section{The combination of vemurafenib with ERK5 pathway inhibitors provides enhanced inhibition of melanoma cell growth compared to single treatments}

The data above demonstrate that BRAF is an upstream activator of ERK5. However, a 24-hour treatment with vemurafenib did not decrease nuclear ERK5 (Fig. 6a), likely due to the occurrence of MEK5-dependent phosphorylation of ERK5 upon MEK1/2 inhibition, as recently reported [36]. In keeping with that, combined treatment with vemurafenib and BIX02189 was needed to reduce the amount of nuclear ERK5 in A375 and SK-Mel-5 melanoma cells (Fig. 6a). Similar effects, i.e., reduction of active nuclear ERK5, were elicited by combination of vemurafenib and XMD8-92 (Supplementary Figure 6). To test whether targeting both the MEK5-ERK5 and BRAF pathway leads to a better response than single treatments, we performed 2D colony formation assays using vemurafenib in combination with XMD8-92 or BIX02189 at IC50 concentrations (Supplementary Figure 7). Either drug combination was more effective than single treatments, preventing colony formation completely (Fig. 6b, c). Likewise, the combination of vemurafenib with XMD8-92 displayed additive effects in reducing proliferation of A375 and SKMel-5 grown in monolayer (Fig. 6d, e). Taken together, these data suggest that vemurafenib and ERK5 pathway inhibitors cooperate in reducing melanoma cell growth.

To examine the efficacy of vemurafenib and XMD8-92 combination in vivo, we assessed the effects of single drugs or their combination in a pre-clinical experimental setting based on BRAFV600E-expressing A375 xenografts. Treatments started when tumors were palpable. Single treatment with low doses of vemurafenib $(20 \mathrm{mg} / \mathrm{kg})[37$, 38] or XMD8-92 (25 mg/kg) [21] twice a day for 19 days produced a $50 \%$ tumor growth inhibition compared to the control group (Fig. 6f, g). Combination treatment with vemurafenib (20 mg/kg) and XMD8-92 (25 mg/kg) achieved a significantly greater antitumor effect than either agent alone (Combo vs Vem $p=0.05$; Combo vs XMD8$92 p=0.005$ ), paralleling results obtained in vitro. Treatment with single agents or their combination was generally well tolerated, without significant weight loss or other overt side effects. Altogether, these results demonstrate that inhibition of the ERK5 pathway improves the effect of vemurafenib treatment against melanoma growth in vitro and in vivo.

\section{Discussion}

In this study, we demonstrate the requirement of the ERK5 pathway for melanoma growth. Indeed, genetic silencing of ERK5 or pharmacological inhibition of ERK5 signaling with two chemically unrelated small molecules dramatically reduce the proliferation of melanoma cells harboring wt or oncogenic BRAF. Importantly, combination of vemurafenib and ERK5 pathway inhibitors, used at doses able to reduce cell proliferation by half, suppresses $2 \mathrm{D}$ colony formation and is more effective than single treatments in reducing growth of BRAFV600E melanoma xenografts. Mechanistically, we show that oncogenic BRAF positively regulates the expression, phosphorylation and nuclear localization of ERK5 as well as its kinase and transcriptional transactivator activities.

Our immunohistochemical analysis shows that ERK5 is expressed at different levels in the majority of human melanomas. In addition, in silico data analysis indicates that $47 \%$ of melanoma patients have alterations in components of the ERK5 pathway. These alterations mainly consist of increased mRNA or gene amplification that are likely responsible for enhanced activation of the pathway. Interestingly, melanoma patients with increased mRNA or amplification of MAPK7, the gene encoding for ERK5, have a shorter disease-free survival compared to patients without such alterations. We also found that 5 out of 479 melanoma patients harbor MAPK7 missense mutations, including P789S and A424S, two potentially phosphorylable sites worth being characterized in future studies. Overall, these data identify a wide subgroup of melanoma patients that might benefit from targeting the ERK5 pathway.

Our data indicate that pharmacological inhibition of the ERK5 pathway strongly reduces melanoma cell growth. In BRAFV600E-expressing cells, MEK5 or ERK5 inhibitors slow down cell cycle progression with the accumulation of cells in G0/G1 phase, likely due to a decreased phosphorylation of $\mathrm{Rb}$, a key regulator of the $\mathrm{G} 1$ to $\mathrm{S}$ phase transition, as previously described [39]. Furthermore, pharmacological inhibition of MEK5 or ERK5 reduces cyclin D1 levels and increases p21 expression as reported in other cell types [39]. In melanoma cells expressing wt BRAF, pharmacological inhibition of ERK5 signaling reduces melanoma cell growth, increasing apoptosis (BIX02189) or blocking cell cycle progression (XMD8-92). Therefore, both BIX02189 and XMD8-92 reduce melanoma cell growth. More importantly, genetic silencing of ERK5 recapitulates, in vitro and in vivo, the effects of pharmacological inhibition of the ERK5 pathway, pointing to the requirement of ERK5 for proliferation of melanoma cells with either wt or BRAFV600E. 


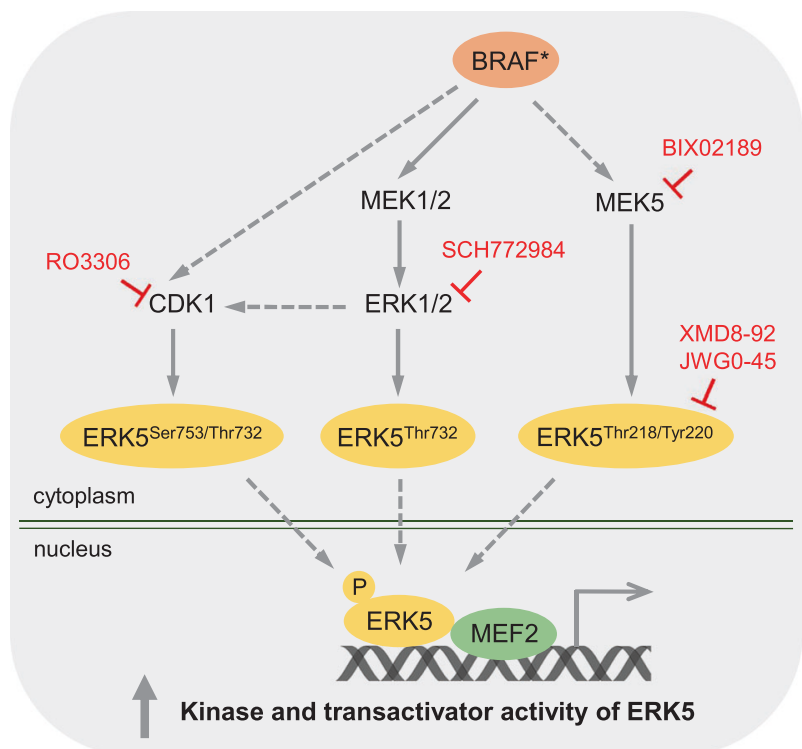

Fig. 7 Proposed mechanism for oncogenic BRAF-dependent ERK5 activation. Oncogenic BRAF may enhance ERK5 phosphorylation and activity through: (i) CDK1 by phosphorylating ERK5 at Ser753 and/or Thr732; (ii) a MEK1/2-ERK1/2-dependent mechanism (Thr732); (iii) a MEK5-dependent mechanism (Thr218/Tyr220). Solid lines indicate direct established regulatory interactions, whereas broken lines illustrate putative interactions. RO-3306 and SCH772984 are, respectively, CDK1 and ERK1/2 inhibitors, XMD8-92 and JWG-045 are ERK5 inhibitors, BIX02189 is a MEK5 inhibitor

An intriguing point emerging from our data is that the involvement of ERK5 in cancer cell proliferation sustained by oncogenic BRAF or RAS is context dependent. Indeed, we show here that ERK5 is required for BRAFV600Edriven proliferation in melanoma in vitro and in vivo. In addition, we previously reported that hepatocellular carcinoma cells, including HepG2 that harbor mutated NRAS (Q61L) [40], are sensitive to genetic and pharmacological inhibition of ERK5 in vitro and in vivo [23]. In contrast, colon cancer cells with KRAS or BRAF mutations do not appear to be addicted to ERK5 activity for proliferation at least in vitro [41].

Another important finding of this study is the identification of a new mechanism of ERK5 regulation (Fig. 7). Our data indicate that oncogenic BRAF increases ERK5 protein level, phosphorylation at several residues and kinase activity. More importantly, BRAFV600E enhances ERK5 nuclear localization, including that in the chromatin-bound fraction, and transcriptional transactivator activity. This is at variance with a previous report showing that BRAFV600E does not enhance ERK5-driven transcriptional activity in presence of overexpressed wt MEK5 in HEK-293T cells [41]. A possible explanation for this discrepancy could be that MEK5 by itself induces an increase of MEF2Dmediated luciferase activity [42], masking the effect of oncogenic BRAF. Alternatively, the effect could be context dependent since our experiments were performed in melanoma cells.

In addition, we show that oncogenic BRAF promotes ERK5 phosphorylation at three crucial sites. First, BRAF increases phosphorylation at Thr732, an event that has been associated with increased ERK5 nuclear localization and ERK5-dependent transcription [35]. Phosphorylation at this residue is prevented by pharmacological inhibition of ERK1/2 and CDK1, pointing to their possible involvement. Second, BRAF enhances ERK5 phosphorylation at Ser753, an additional CDK1 target residue. However, the fact that pharmacological inhibition of CDK1 does not completely abolish phosphorylation at this site leaves open the possibility that Ser753 is either autophosphorylated, as is the case for other residues at C terminus of ERK5 [35, 42], or is the substrate of a yet unidentified kinase. The effect of the ERK1/2 inhibitor SCH772984 in abrogating phosphorylation at Ser753 may indicate that ERK1/2 acts upstream of CDK1, which is consistent with the presence of active CDK1 in oncogenic BRAF-overexpressing cells (Supplementary Figure 5). Finally, overexpression of BRAF induces ERK5 phosphorylation at Thr218/Tyr220, suggesting that MEK5 participates in BRAF-induced ERK5 activation (Fig. 7). Increased ERK5 phosphorylation at Thr218/ Tyr220 by oncogenic BRAF has been showed in a previous report, although the authors reached different conclusions stating that oncogenic BRAF does not stimulate ERK5 phosphorylation [41]. Together, these data indicate that oncogenic BRAF, via CDK1, ERK1/2 and/or MEK5, transduces mitogenic signals to the nucleus through ERK5 (Fig. 7). It has been recently reported that dietary-fat-fueled ketogenesis promotes BRAFV600E melanoma growth [43]. On that basis, it would be interesting to investigate the role of ERK5 in the effect exerted by high fat metabolism on melanoma growth.

Our data indicate that vemurafenib inhibits ERK5 kinase activity, but is not able to reduce the level of nuclear ERK5. The reduction of total and/or active ERK5 is achieved only by vemurafenib together with BIX02189 or XMD8-92, thus providing the rationale for a combined treatment. Indeed, combination of IC50 doses of XMD8-92 and vemurafenib abolished two-dimensional colony formation and drastically reduced melanoma xenograft growth. The experiments presented here suggest that the combination of ERK5 pathway inhibitors with vemurafenib could be a good strategy for the treatment of BRAF-mutant melanoma patients. The combination therapy we propose could be also important to prevent the recently reported ERK5-mediated resistance to vemurafenib-trametinib treatment in melanoma [44]. Further advocating combination therapy targeting MEK1/2-ERK1/2 and ERK5 pathway, a recent report suggested that pharmacological inhibition of ERK1/2 may 
induce ERK5 phosphorylation at MEK5 consensus sites in colon cancer cells [36].

Overall this study suggests that targeting ERK5 might be regarded as first-line therapeutic approach for melanoma patients with wt or oncogenic BRAF, or as a potential therapeutic strategy aimed at preventing resistance to BRAF-MEK1/2 inhibitors. Because the immune system plays a critical role in the progression of melanoma [8], a potential caveat is that systemic inhibition of ERK5 might impact on the immune system functions with respect to tumor progression. Indeed, ERK5 has been reported to either inhibit or stimulate $\mathrm{T}$ cell-mediated antitumor immunity [45, 46] and to be critical for macrophage proliferation [12]. Therefore, in view of a future use of ERK5 pathway inhibitors in human melanoma, the effects of systemic ERK5 inhibition on immune response should be further investigated.

\section{Material and methods}

\section{Cell lines and melanoma samples}

A375 (CRL-1619), MeWo (HTB-65), and HEK-293T (CRL-3216) cells were obtained from ATCC (Manassas, VA, www.lgcstandards-atcc.org). SK-Mel-2, SK-Mel-5, SK-Mel-28, and 501-Mel melanoma cells were kindly provided by Dr. Laura Poliseno (CRL-ITT, Pisa, Italy). Patient-derived SSM2c and M26c melanoma cells were already described [30] (Supplementary Table 2) and were obtained from human melanoma samples after approved protocols by the Ethics Committee. Cell lines were authenticated by cell profiling (Promega PowerPlex Fusion System kit; BMR Genomics s.r.l; Padova, Italy) once a year. Mycoplasma was periodically tested by 4',6-diamidino-2-phenylindole (DAPI) inspection and PCR upon thawing of a new batch of cells and once a month. Cultures are renewed every 2 months.

\section{Drugs}

The following drugs were used: ERK5 inhibitors XMD8-92 [31] and JWG-045 [34] have been developed in Gray's laboratory; MEK5 inhibitor BIX02189 [32], ERK1/2 inhibitor SCH772984 [47] and BRAFV600E inhibitor vemurafenib [48] (Selleckchem, Italy, www.selleckchem.com); CDK1 inhibitor RO-3306 (MedChem Express, www. medchemexpress.com) [49].

\section{RNA interference}

Lentiviruses were produced in HEK-293T cells as previously reported [23]. Lentiviral vectors for stable knockdown of ERK5 in melanoma cells were TRC1.5pLKO.1-puro vector containing non targeting sequence shRNA (LV-c), targeting human MAPK7 (NM_139032, NM_139034, NM_002749, NM_139033) clone ID: TRCN0000010275 (LV-shERK5-1) as described [23] and clone ID:TRCN0000010271 (5'-CCGGCCAGTCCAACCTACCAGTCCTCTCGAGAGGACTGGTAGGTTGGACTGGTTTTT-3'; LV-shERK5-2). Transduced cells were selected with $2 \mu \mathrm{g} / \mathrm{ml}$ puromycin for at least $72 \mathrm{~h}$.

\section{Measurement of cell viability and cell cycle phase distribution}

The number of viable cells in culture was evaluated by counting trypan blue-negative cells at the indicated timepoints with an hemocytometer. IC50 values were calculated using GraphPad Prism software. Cell cycle phase distribution (propidium iodide staining) was estimated by flow cytometry using a FACSCanto (Beckton \& Dickinson, San Josè, CA, USA) as previously reported [23].

\section{D clonogenic assay}

For colony formation assay, 500 (A375) or 3500 (SK-MEL5) cells were seeded in p60 dishes in the presence of drugs or vehicle (DMSO). Colonies (with more than 50 cells, i.e., 8 cell diameter) were counted following crystal violet staining after 7 (A375) or 14 (SK-MEL-5) days [50]. IC50 values were calculated using GraphPad Prism software.

\section{Cell lysis and Western blotting}

Cells were lysed as already described in Laemmli [12] or RIPA buffer [30]. Nucleo-cytoplasm fractions have been obtained as previously described [23]. In other experiments, subcellular fractionation was performed using the subcellular protein fractionation kit (Pierce, Thermo Fisher Scientific, Waltham, MA, USA) according to manufacturer's instructions. Proteins were separated by SDS-PAGE and transferred into PVDF or nitrocellulose membranes (Millipore, www.merckmillipore.com) by electroblotting. Infrared imaging (Odyssey, Licor, www. licor.com) or chemiluminescent detection were performed. Images were recorded as TIFF files for quantification with Adobe Photoshop software. Antibodies used are listed in Supplementary Table 3.

\section{ERK5 kinase assay}

Kinase activity of endogenous ERK5 was measured using a non-radioactive ERK Assay Kit (\#17-191, Merck Millipore, Billerica, MA, USA) following manufacturer's instruction. The ability of immunoprecipitated ERK5 to phosphorylate 
myelin basic protein (MBP) was evaluated by western blotting.

\section{Luciferase assay}

Luciferase reporter (3XMEF2-luc plasmid \# 32967 was a gift from Ron Prywes, Addgene, Teddington, UK) was used in combination with Renilla luciferase pRL-TK reporter vector (Promega, Madison, WI) to normalize luciferase activities; pcDNA vector was used to equal DNA amounts. Luminescence was measured using the Dual-Glo Luciferase Assay System (Promega) and the GloMax ${ }^{\circledR}$ 20/20 Luminometer (Promega).

\section{Xenografts}

In the first set of experiments, A375 and SSM2c melanoma cells transduced with LV-c or LV-shERK5 were resuspended in Matrigel (Corning, www.corning.com)/DMEM (1/1) and subcutaneously injected (10,000 cells/injection) into lateral flanks of adult ( 8 weeks) female athymic nude mice (Foxn1 nu/nu) (Harlan Laboratories, Udine, Italy). Number of animals (sample size) per group, calculated on the basis of http://www.biomath.info/power/ttest.htm (two groups $>t$-test on group means $>$ find sample size), was 6 for A375 and SSM2c LV-shERK5 and 7 for SSM2c LV-c.

In the second set of experiments, parental A375 cells were subcutaneously injected as above. Once tumors were palpable $\left(4 \mathrm{~mm}^{3}\right)$, blind randomization of mice in four groups of nine mice each was performed on the basis of size and presence/absence of palpable tumors on each flank. Mice were treated intraperitoneally twice a day for 19 days with vehicle (30\% 2-hydroxypropyl- $\beta$-cyclodextrin), XMD8-92 (25 mg/kg), vemurafenib (20 mg/kg), or a combination of both drugs. In both experiments subcutaneous tumor size was measured three times a week with a caliper. Tumor volumes were calculated using the formula: $V=$ $W^{2} \times L \times 0.5$, where $W$ and $L$ are tumor width and length, respectively. On the basis of pre-established criteria, tumors that had not developed at the time of randomization were excluded from the analysis.

The experiments were approved by the Italian Ministry of Health (authorization no. 213/2015-PR) and were in accordance with the Italian guidelines and regulations.

\section{Plasmids and transfection}

pcDNA3.1-BRAFV600E and pcDNA3.1-BRAFwt constructs were a kind gift from Laura Poliseno (CRL-ITT, Pisa, Italy). pcDNA3.1-HA-ERK5wt construct was a kind gift from Atanasio Pandiella (CIC, Salamanca, Spain). The pcMV5-MEK5DD-HA (a constitutively active form of MEK5) was generously provided by Jiing-Dwan Lee
(Scripps Institute, La Jolla, CA, USA). HEK-293T or M26c cells were plated on six-well dishes $\left(3 \times 10^{5}\right.$ cells/well $)$ and transfected after $24 \mathrm{~h}$ with a total amount of $3 \mu \mathrm{g}$ of plasmid DNA using Lipofectamin2000 (Invitrogen, Thermo Fisher Scientific), following manufacturer's instructions. Cells were lysed after $24-48 \mathrm{~h}$. Drugs were added $18 \mathrm{~h}$ before lysis.

\section{Immunohistochemistry}

Immunohistochemistry was performed on tissue microarrays (US Biomax, Inc., http://www.biomax.us) of formalin-fixed paraffin-embedded specimens of human nevi and melanomas. After citrate buffer antigen retrieval, staining was performed with the UltraVision LP Detection System HRP Polymer kit (\#TL-015-HD, Thermo Fisher Scientific) following manufacturer's instructions. Sections were incubated overnight at $4{ }^{\circ} \mathrm{C}$ with mouse monoclonal anti-ERK5 (C-7) (sc-398015, 1:100 dilution) (S. Cruz, CA, USA). DAB (3,3'-diaminobenzidine) (Thermo Fisher Scientific) or AEC (3-amino-9-ethylcarbazole)(\#K3461, Dako, Copenhagen, Denmark) were used as chromogens. Sections stained with AEC were counterstained with hematoxylin.

\section{Statistical analysis}

Data represent mean \pm SEM or mean \pm SD values calculated on at least three independent experiments. The exact number of experiments performed and used for statistical analysis is indicated in each figure legend. No statistical methods were used for sample size selection. Variance between groups that have been statistically compared was similar. $P$ values were calculated using Student's $t$-test (two groups) or one-way analysis of variance (more than two groups; multiple comparison using Bonferroni's correction). Analysis of in vivo combined treatments were performed using the false discovery rate adjustment for multiple comparisons. A two-tailed value of $p<0.05$ was considered statistically significant. Number of mice used for each experiment were indicated above.

Acknowledgements This work was supported by a grant from Associazione Italiana per la Ricerca sul Cancro, AIRC, (IG-15282 to ER and IG-14184 to BS). We are grateful to Dr P. Dello Sbarba (University of Florence, Florence, Italy) and to Dr Chiariello (Istituto Toscano Tumori, Siena, Italy) for critical revision of the manuscript. We also thank Dr A. Pandiella for providing antibodies and Dr L. Poliseno (Istituto Toscano Tumori, Pisa, Italy) for providing melanoma cells and plasmids.

Author contributions Conception and design: E.R. and B.S. Development of methodology: I.T., S.P., A.T., S.G., E.R., and B.S. Acquisition of data: I.T., S.P., A.T., S.G., E.R., and B.S. Analysis and interpretation of data: E.R., B.S.; I.T., S.P., A.T., S.G., and C.U. Writing: E.R. and B.S. Review and/or revision of the manuscript: E.R., B.S., I.T., S.P., S.G., and A.T. Administrative, technical, or material 
support: E.R., B.S., and I.T. Study supervision: E.R. and B.S. Provision of melanoma samples and clinical information: L.B. Assessment of immunohistochemical staining: C.U. Provision of drugs and competences for their use: N.S.G., X.D., and J.W.

\section{Compliance with Ethical Standards}

Conflict of interest Dana Farber Cancer Institute, the employer of NSG, holds the right of the compound XMD8-92 and JWG-045 used in the present study. The authors declare that they have no conflict of interest.

Open Access This article is licensed under a Creative Commons Attribution-NonCommercial-ShareAlike 4.0 International License, which permits any non-commercial use, sharing, adaptation, distribution and reproduction in any medium or format, as long as you give appropriate credit to the original author(s) and the source, provide a link to the Creative Commons license, and indicate if changes were made. If you remix, transform, or build upon this article or a part thereof, you must distribute your contributions under the same license as the original. The images or other third party material in this article are included in the article's Creative Commons license, unless indicated otherwise in a credit line to the material. If material is not included in the article's Creative Commons license and your intended use is not permitted by statutory regulation or exceeds the permitted use, you will need to obtain permission directly from the copyright holder. To view a copy of this license, visit http://creativecommons. org/licenses/by-nc-sa/4.0/.

\section{References}

1. Balch CM, Gershenwald JE, Soong SJ, Thompson JF, Atkins MB, Byrd DR, et al. Final version of 2009 AJCC melanoma staging and classification. J Clin Oncol. 2009;27:6199-6106.

2. Lo JA, Fisher DE. The melanoma revolution: from UV carcinogenesis to a new era in therapeutics. Science. 2014;346:945-9.

3. Cancer Genome Atlas Research Network. Genomic classification of cutaneous melanoma. Cell. 2015;161:1681-96.

4. Krauthammer M, Kong Y, Bacchiocchi A, Evans P, Pornputtapong $\mathrm{N}, \mathrm{Wu} \mathrm{C}$, et al. Exome sequencing identifies recurrent mutations in NF1 and RASopathy genes in sun-exposed melanomas. Nat Genet. 2015;47:996-1002.

5. Cohen C, Zavala-Pompa A, Sequeira JH, Shoji M, Sexton DG, Cotsonis G, et al. Mitogen-actived protein kinase activation is an early event in melanoma progression. Clin Cancer Res. 2002;8:3728-33.

6. Chapman PB, Hauschild A, Robert C, Haanen JB, Ascierto P, Larkin J, et al. Improved survival with vemurafenib in melanoma with BRAF V600E mutation. N Engl J Med. 2011;364:2507-16.

7. Flaherty KT, Infante JR, Daud A, Gonzalez R, Kefford RF, Sosman J, et al. Combined BRAF and MEK inhibition in melanoma with BRAF V600 mutations. $N$ Engl J Med. 2012;367:1694-1603.

8. Larkin J, Hodi FS, Wolchok JD. Combined nivolumab and ipilimumab or monotherapy in untreated melanoma. N Engl J Med. 2015;373:23-34.

9. Samatar AA, Poulikakos PI. Targeting RAS-ERK signalling in cancer: promises and challenges. Nat Rev Drug Discov. 2014;13:928-42.

10. Teixidó C, González-Cao M, Karachaliou N, Rosell R. Predictive factors for immunotherapy in melanoma. Ann Transl Med. 2015;3:208.

11. Drew BA, Burow ME, Beckman BS. MEK5/ERK5 pathway: the first fifteen years. Biochim Biophys Acta. 2012;1825:37-48.
12. Rovida E, Spinelli E, Sdelci S, Barbetti V, Morandi A, Giuntoli S, et al. ERK5/BMK1 is indispensable for optimal colonystimulating factor 1 (CSF-1) induced proliferation in macrophages in a Src-dependent fashion. J Immunol. 2008; 180:4166-72.

13. Esparís-Ogando A, Díaz-Rodríguez E, Montero JC, Yuste L, Crespo P, Pandiella A. Erk5 participates in neuregulin signal transduction and is constitutively active in breast cancer cells overexpressing ErbB2. Mol Cell Biol. 2002;22:270-85.

14. Nithianandarajah-Jones GN, Wilm B, Goldring GE, Müller J, Cross MJ. ERK5: structure, regulation and function. Cell Signal. 2012;24:2187-96.

15. Díaz-Rodríguez E, Pandiella A. Multisite phosphorylation of Erk5 in mitosis. J Cell Sci. 2010;123:3146-56.

16. Iñesta-Vaquera FA, Campbell DG, Tournier C, Gómez N, Lizcano JM, Cuenda A. Alternative ERK5 regulation by phosphorylation during the cell cycle. Cell Signal. 2010;22:1829-37.

17. Raviv Z, Kalie E, Seger R. MEK5 and ERK5 are localized in the nuclei of resting as well as stimulated cells, while MEKK2 translocates from the cytosol to the nucleus upon stimulation. $\mathrm{J}$ Cell Sci. 2004;117:1773-84.

18. Buschbeck M, Ullrich A. The unique C-terminal tail of the mitogen-activated protein kinase ERK5 regulates its activation and nuclear shuttling. J Biol Chem. 2005;280:2659-67.

19. Gomez N, Erazo T, Lizcano JM. ERK5 and cell proliferation: nuclear localization is what matters. Front Cell Dev Biol. 2016;4:105.

20. Erazo T, Moreno A, Ruiz-Babot G, Rodríguez-Asiain A, Morrice NA, Espadamala $J$, et al. Canonical and kinase activityindependent mechanisms for extracellular signal-regulated kinase 5 (ERK5) nuclear translocation require dissociation of Hsp90 from the ERK5-Cdc37 complex. Mol Cell Biol. 2013;33:1671-86

21. Al-Ejeh F, Miranda M, Shi W, Simpson PT, Song S, Vargas AC, et al. Kinome profiling reveals breast cancer heterogeneity and identifies targeted therapeutic opportunities for triple negative breast cancer. Oncotarget. 2014;5:3145-58.

22. McCracken SR, Ramsay A, Heer R, Mathers ME, Jenkins BL, Edwards $\mathrm{J}$, et al. Aberrant expression of extracellular signalregulated kinase 5 in human prostate cancer. Oncogene. 2008;27:2978-88.

23. Rovida E, Di Maira G, Tusa I, Cannito S, Paternostro C, Navari N, et al. The mitogen-activated protein kinase ERK5 regulates the development and growth of hepatocellular carcinoma. Gut. 2015;64:1454-65.

24. Carvajal-Vergara X, Tabera S, Montero JC, Esparís-Ogando A, López-Pérez R, Mateo G, et al. Multifunctional role of Erk5 in multiple myeloma. Blood. 2005;105:4492-9.

25. Yang Q, Lee JD. Targeting the BMK1 MAP kinase pathway in cancer therapy. Clin Cancer Res. 2011;17:3527-32.

26. Simões AE, Rodrigues CM, Borralho PM. The MEK5/ ERK5 signalling pathway in cancer: a promising novel therapeutic target. Drug Discov Today. 2016;21:1654-63.

27. Kato Y, Zhao M, Morikawa A, Sugiyama T, Chakravortty D, Koide $\mathrm{N}$, et al. Big mitogen-activated kinase regulates multiple members of the MEF2 protein family. J Biol Chem. 2000;275:18534-40.

28. Cerami E, Gao J, Dogrusoz U, Gross BE, Sumer SO, Aksoy BA, et al. The cBio cancer genomics portal: an open platform for exploring multidimensional cancer genomics data. Cancer Discov. 2012;2:401-4.

29. Gao J, Aksoy BA, Dogrusoz U, Dresdner G, Gross B, Sumer SO, et al. Integrative analysis of complex cancer genomics and clinical profiles using the cBioPortal. Sci Signal. 2013;6:11.

30. Santini R, Pietrobono S, Pandolfi S, Montagnani V, D'Amico M, Penachioni JY, et al. SOX2 regulates self-renewal and 
tumorigenicity of human melanoma-initiating cells. Oncogene. 2014;33:4697-4608.

31. Yang Q, Deng X, Lu B, Cameron M, Fearns C, Patricelli MP, et al. Pharmacological inhibition of BMK1 suppresses tumor growth through promyelocytic leukemia protein. Cancer Cell. 2010;18:258-67.

32. Tatake RJ, O'Neill MM, Kennedy CA, Wayne AL, Jakes S, Wu $\mathrm{D}$, et al. Identification of pharmacological inhibitors of the MEK5/ ERK5 pathway. Biochem Biophys Res Commun. 2008;377:120-5.

33. Lin EC, Amantea CM, Nomanbhoy TK, Weissig H, Ishiyama J, $\mathrm{Hu} \mathrm{Y}$, et al. ERK5 kinase activity is dispensable for cellular immune response and proliferation. Proc Natl Acad Sci USA. 2016;113:11865-70.

34. Williams CA, Fernandez-Alonso R, Wang J, Toth R, Gray NS, Findlay GM. Erk5 is a key regulator of naive-primed transition and embryonic stem cell identity. Cell Rep. 2016;16:1820-8.

35. Honda T, Obara Y, Yamauchi A, Couvillon AD, Mason JJ, Ishii K, et al. Phosphorylation of ERK5 on Thr732 is associated with ERK5 nuclear localization and ERK5-dependent transcription. PLoS ONE. 2015;10:e0117914.

36. de Jong PR, Taniguchi K, Harris AR, Bertin S, Takahashi N, Duong $\mathrm{J}$, et al. ERK5 signalling rescues intestinal epithelial turnover and tumour cell proliferation upon ERK1/2 abrogation. Nat Commun. 2016;7:11551.

37. Yang H, Higgins B, Kolinsky K, Packman K, Go Z, Iyer R, et al. RG7204 (PLX4032), a selective BRAFV600E inhibitor, displays potent antitumor activity in preclinical melanoma models. Cancer Res. 2010;70:5518-27.

38. Paoluzzi L, Hanniford D, Sokolova E, Osman I, Darvishian F, Wang J, et al. BET and BRAF inhibitors act synergistically against BRAF-mutant melanoma. Cancer Med. 2016;5:1183-93.

39. Perez-Madrigal D, Finegan KG, Paramo B, Tournier C. The extracellular-regulated protein kinase 5 (ERK5) promotes cell proliferation through the down-regulation of inhibitors of cyclin dependent protein kinases (CDKs). Cell Signal. 2012;24:2360-8.
40. Davies H, Bignell GR, Cox C, Stephens P, Edkins S, Clegg S, et al. Mutations of the BRAF gene in human cancer. Nature. 2002;417:949-54.

41. Lochhead PA, Clark J, Wang LZ, Gilmour L, Squires M, Gilley $\mathrm{R}$, et al. Tumor cells with KRAS or BRAF mutations or ERK5/ MAPK7 amplification are not addicted to ERK5 activity for cell proliferation. Cell Cycle. 2016;15:506-18.

42. Morimoto H, Kondoh K, Nishimoto S, Terasawa K, Nishida E. Activation of a C-terminal transcriptional activation domain of ERK5 by autophosphorylation. J Biol Chem. 2007;282:35449-56.

43. Xia S, Lin R, Jin L, Zhao L, Kang HB, Pan Y, et al. Prevention of dietary-fat-fueled ketogenesis attenuates BRAF V600E tumor growth. Cell Metab. 2017;25:358-73.

44. Song C, Wang L, Xu Q, Wang K, Xie D, Yu Z, et al. Targeting BMK1 impairs the drug resistance to combined inhibition of BRAF and MEK1/2 in melanoma. Sci Rep. 2017;7:46244.

45. Loveridge CJ, Mui EJ, Patel R, Tan EH, Ahmad I, Welsh M, Galbraith J, et al. Increased T-cell infiltration elicited by Erk5 deletion in a Pten-deficient mouse model of prostate carcinogenesis. Cancer Res. 2017;15(77):3158-68.

46. Rovira-Clavé X, Angulo-Ibáñez M, Tournier C, Reina M, Espel E. Dual role of ERK5 in the regulation of $\mathrm{T}$ cell receptor expression at the T cell surface. J Leukoc Biol. 2016;99:143-52.

47. Morris EJ, Jha S, Restaino CR, Dayananth P, Zhu H, Cooper A, et al. Discovery of a novel ERK inhibitor with activity in models of acquired resistance to BRAF and MEK inhibitors. Cancer Discov. 2013;3:742-50.

48. Sala E, Mologni L, Truffa S, Gaetano C, Bollag GE, GambacortiPasserini C. BRAF silencing by short hairpin RNA or chemical blockade by PLX4032 leads to different responses in melanoma and thyroid carcinoma cells. Mol Cancer Res. 2008;6:751-9.

49. Vassilev LT, Tovar C, Chen S, Knezevic D, Zhao X, Sun H, et al. Selective small-molecule inhibitor reveals critical mitotic functions of human CDK1. Proc Natl Acad Sci USA. 2006;103:10660-5.

50. Franken NA, Rodermond HM, Stap J, Haveman J, van Bree C. Clonogenic assay of cells in vitro. Nat Protoc. 2006;1:2315-9. 